\title{
Role of Domiciliary Noninvasive Ventilation in Chronic Obstructive Pulmonary Disease Patients Requiring Repeated Admissions with Acute Type II Respiratory Failure: A Prospective Cohort Study
}

\author{
K. P. Suraj, E. Jyothi, R. Rakhi \\ Department of Pulmonary Medicine, Government Medical College, Kozhikode, Kerala, India
}

\section{Abstract}

Background: Acute exacerbation of chronic obstructive pulmonary disease (AECOPD) with acute hypercapnic respiratory failure (AHRF) is associated with high mortality and increased risk for further exacerbations and hospitalization. While there is ample evidence regarding the benefit of noninvasive ventilation (NIV) during AECOPD, evidence supporting long-term noninvasive ventilation (LTNIV) for more stable COPD patients is limited. Objective: The aim of this study is to assess the effectiveness of LTNIV in COPD patients requiring frequent hospital admissions and NIV support for AHRF. Materials and Methods: A prospective cohort study including 120 patients having survived an admission requiring NIV support for AHRF due to COPD, with a history of $\geq 3$ similar episodes in the past year. Patients were advised LTNIV (30) with standard treatment, or (90) standard treatment alone. Both groups were followed up for 1 year. Among non-NIV group 10 died, and 8 lost follow-up, whereas two died in NIV group. The primary endpoint was death. Data of remaining 100 patients were analyzed for other objectives-number of readmissions, AHRF, Intensive Care Unit (ICU)/ventilator requirement, dyspnea, quality of life, exercise tolerance, lung function, and arterial blood gases. Results: LTNIV group had 40\% reduction in mortality (6.6\% vs. 11.1\%). There was significant reduction in number of hospital admissions (28.6\% vs. $84.7 \%: P<0.05)$, ICU admissions $(7.1 \%$ vs. $56.9 \%: P=0.01)$, ventilator requirement $(3.6 \%$ vs. $30.6 \%$ : $P=0.003)$, AHRF $(7.1 \%$ vs. $48.6 \%: P=0.000)$ and improvement in partial arterial $\mathrm{CO}_{2}$ pressure $(39.8 \pm 2.1$ vs. $57.03 \pm 3.7 \mathrm{mmHg})$ and severe respiratory insufficiency score $(P<0.05)$ among LTNIV group, but no significant change in lung function and exercise tolerance. Conclusion: Patients tolerated LTNIV well and had a better outcome compared to those without NIV. LTNIV may be considered in patients with recurrent AHRF.

Keywords: Acute exacerbation of chronic obstructive pulmonary disease, home mechanical ventilation, hypercapnic respiratory failure, long-term noninvasive ventilation, noninvasive ventilation

\section{INTRODUCTION}

Chronic obstructive pulmonary disease (COPD) is the $4^{\text {th }}$ leading cause of death in the world and is projected to be the $3^{\text {rd }}$ leading cause of death by 2020 as per GOLD 2017. Globally, the prevalence of COPD is alarmingly rising in countries of all levels of development. Hence in the arena of management, more importance is being given to modify the disease process, to provide better medical care and thus reduce the burden on patients, caregivers, and society. Main factors that determine the prognosis include frequency of exacerbations and respiratory failure, which may or may not require ventilator assistance ${ }^{[1]}$ Advances in technology have

\begin{tabular}{|l|l|}
\hline \multicolumn{2}{|c|}{ Access this article online } \\
\hline Quick Response Code: & Website: \\
\hline & www.ijccm.org \\
\hline & \\
\hline
\end{tabular}

revolutionized mechanical ventilation. Over the past three decades, the application of noninvasive ventilation (NIV) has emerged as a core therapy in the management of patients with acute and chronic respiratory failure. ${ }^{[2]}$ It has become an integral tool in the management of respiratory failure, in both home setting and the critical care units. ${ }^{[3]}$

$$
\begin{array}{r}
\text { Address for correspondence: Dr. E. Jyothi, } \\
\text { Department of Pulmonary Medicine, Government Medical College, } \\
\text { Kozhikode, Kerala, India. } \\
\text { E-mail: drjyothie@yahoo.com }
\end{array}
$$

This is an open access journal, and articles are distributed under the terms of the Creative Commons Attribution-NonCommercial-ShareAlike 4.0 License, which allows others to remix, tweak, and build upon the work non-commercially, as long as appropriate credit is given and the new creations are licensed under the identical terms.

For reprints contact: reprints@medknow.com

How to cite this article: Suraj KP, Jyothi E, Rakhi R. Role of domiciliary noninvasive ventilation in chronic obstructive pulmonary disease patients requiring repeated admissions with acute Type II respiratory failure: A prospective cohort study. Indian J Crit Care Med 2018;22:397-401. 
Despite the success of NIV in the management of acute hypercapnic respiratory failure (AHRF) in COPD patients, survivors in this group have a high risk of further life-threatening events. Previous studies on the benefits of home NIV in patients with COPD are conflicting. While there is ample evidence regarding the benefit of NIV during an acute exacerbation of COPD, evidence supporting long-term noninvasive ventilation (LTNIV) for more stable COPD patients is limited. This study is aimed to assess the effectiveness of LTNIV in COPD patients requiring frequent hospital admissions and NIV support for AHRF.

\section{Materials and Methods}

This prospective cohort study was conducted at a tertiary care center in North Kerala and patients were recruited from September 2015 to November 2016. We screened COPD patients admitted in our department with severe exacerbation and persistent respiratory acidosis (indicated by an arterial $\mathrm{pH}<7.35$ and partial arterial $\mathrm{CO}_{2}$ pressure $\left.\left(\mathrm{PaCO}_{2}\right)>45 \mathrm{mmHg}\right)$ despite initial treatment with bronchodilators, corticosteroids \pm antibiotics, $z$ requiring NIV/ventilatory support with history of $\geq 3$ similar episodes (including present episode) in the past 1 year, who were under optimal medical treatment (tiotropium $9 \mu \mathrm{g}+$ fomoterol $6 \mu \mathrm{g}$ metered-dose inhaler (MDI) 2 puffs morning and budesonide $200 \mu \mathrm{g}+$ formoterol $6 \mu \mathrm{g}$ MDI 2 puffs at night, theophylline, vaccinated with pneumococcal and influenza vaccine). The study population included those who survived the present episode of $\mathrm{AHRF}$ and $\mathrm{PaCO}_{2}$ remained $>45 \mathrm{mmHg}$ at the time of discharge. Arterial blood gas (ABG) analysis for inclusion purpose was done $6 \mathrm{~h}$ after stopping NIV support. Those with coexisting obstructive sleep apnea/obesity hypoventilation syndrome, chest wall or neuromuscular disease, significant systemic illness, and moribund patients were excluded from the study.

A total of 120 patients admitted with AHRF met the inclusion criteria. All the patients were advised long-term NIV on discharge. Patients and a close relative were counseled in detail about benefits and problems of LTNIV use. Only 30 patients were willing for home NIV for financial and social reasons. The remaining 90 patients receiving only standard treatment were taken as controls [Flow chart 1]. All the patients were followed up for 1 year.

Patients were instructed to use NIV while in bed and intermittently for $2 \mathrm{~h}$ while awake ( $1 \mathrm{~h}$ during forenoon and $1 \mathrm{~h}$ during the afternoon) during $1^{\text {st }}$ month using an oronasal mask. After that, patients were instructed to use NIV for a minimum of $6 \mathrm{~h}$ every night. Pressure setting advised was $1 \mathrm{~cm}$ of $\mathrm{H}_{2} \mathrm{O}$ less than the pressure with which the patient was managed at the time of discharge. Adequate instructions regarding how to use NIV machine, service center contacts for any emergency were given to the patient. These patients were assessed 1 month, 6 months and 1 year on follow-up. Baseline 6 min walk test (6MWT) and spirometry were done during the

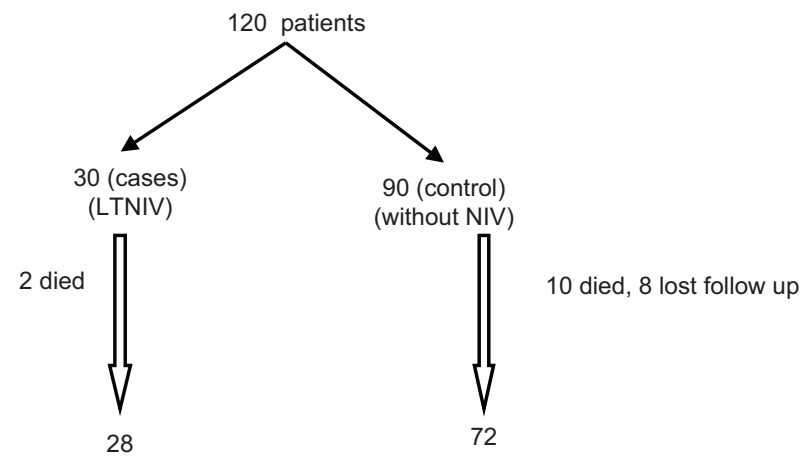

Flowchart 1: Flow chart showing patient recruitment, allocation into NIV and non NIV group and patients available for follow-up analysis

$1^{\text {st }}$ month visit as patients were not fit to perform these tests at the time of discharge. ABG and 6MWT were repeated in the follow-up visits. Spirometry was repeated during the final visit. Compliance was assessed during review visits using questionnaire and details collected over the phone, at the $4^{\text {th }}, 8^{\text {th }}$, and $10^{\text {th }}$ months. Instructions regarding the use of saline nasal drops as needed and adequate hydration to relieve drying of the nose, pressure relieving dressings on the bridge of the nose to prevent ulceration and effective mouth care, were also given.

Death was the primary endpoint of the study. Secondary endpoints included some readmissions, AHRF, Intensive Care Unit (ICU)/ventilator requirement, quality of life, exercise tolerance, lung function, and ABGs. Quality of life was assessed using severe respiratory insufficiency (SRI) questionnaire.

Written informed consent was obtained from all patients, and the study protocol was approved by the Institutional Research and Ethical Committee. Statistical analysis was performed using SPSS version 18 software. Differences were measured using Chi-square test and $t$-test. Results are presented as mean with standard deviation. A value of $P<0.05$ was considered as statistically significant.

\section{Observations}

Males constituted majority of the study population - $71.4 \%$ (NIV group), 59.7\% (non-NIV group). Mean age of the NIV group was $56.8 \pm 4.1$ and control group was $59.8 \pm 3.2$ years. The mean inspiratory positive airway pressure prescribed was $15.4 \mathrm{~cm}$ of $\mathrm{H}_{2} \mathrm{O}$ (range $12-18 \mathrm{~cm}$ of $\mathrm{H}_{2} \mathrm{O}$ ) and mean expiratory positive airway pressure (EPAP) was $7.4 \mathrm{~cm}$ of $\mathrm{H}_{2} \mathrm{O}$ (range 5-9 $\mathrm{cm}$ of $\mathrm{H}_{2} \mathrm{O}$ ). Baseline values are depicted in Table 1.

Mortality among the NIV group was $6.6 \%$ (2 out of 30 ) and non-NIV group was $11.1 \%$ (10 out of 90) (relative risk - 0.6) revealing a $40 \%$ reduction in mortality among those using home NIV. NIV group revealed lesser number of hospital admissions (28.6\% vs. 84.7\%), ICU admissions (7.1\% vs. $56.9 \%)$, ventilator requirement (3.6\% vs. $30.6 \%)$, and AHRF (7.1\% vs. 48.6\%) [Table 2].

ABG among the two groups at the end of the study showed statistically significant improvement in NIV group 
Suraj, et al.: Role of domiciliary noninvasive ventilation in chronic obstructive pulmonary disease patients requiring repeated admissions with acute type ii respiratory failure: A prospective cohort study

[Figures 1and 2]. Patients using home NIV revealed the better quality of life compared to non-NIV group [Table 3].

However, we could not reveal significant improvement in lung function and exercise tolerance [Table 4].

Compliance with NIV use was good in the study group. The average nocturnal use of NIV was $>5 \mathrm{~h}$ per night. Regarding the adverse effects, 10 out of 28 patients with NIV reported minor adverse effects-abdominal distension (5), nasal drying (2), and nasal bridge ulceration (3). Pressure area assessment, skin care, and oral hygiene were assessed at each review. None of the patients had significant adverse effects requiring discontinuation of NIV.

\section{Discussion}

Owing to its significant burden both on the individual and society, COPD is an area of intensive epidemiologic and

\section{Table 1: Baseline values}

\begin{tabular}{lcc}
\hline & NIV group & Non NIV group \\
\hline Males & $71.4 \%(21)$ & $59.7 \%(56)$ \\
Females & $28.6 \%(9)$ & $40.3 \%(34)$ \\
Age $(\mathrm{yrs})$ & $56.8 \pm 4.1$ & $59.8 \pm 3.2$ \\
$\mathrm{PaCO}_{2}(\mathrm{mmHg})$ & $49.2 \pm 1.9$ & $49.1 \pm 2.1$ \\
$\mathrm{PaO}_{2}(\mathrm{mmHg})$ & $59.3 \pm 3.1$ & $58.9 \pm 2.8$ \\
\hline
\end{tabular}

Table 2: Comparison of outcome between patients on noninvasive ventilation and controls

\begin{tabular}{lccc}
\hline & NIV group & Non NIV group & \\
\hline Mortality & $6.6 \%(2)$ & $11.1 \%(10)$ & $\mathrm{RR}=0.6$ \\
Hospital admissions & $28.6 \%(8)$ & $84.7 \%(61)$ & $P=0.002$ \\
ICU admissions & $7.1 \%(2)$ & $56.9 \%(41)$ & $P=0.003$ \\
Ventilator requirement & $3.6 \%(1)$ & $30.6 \%(22)$ & $P=0.002$ \\
AHRF & $7.1 \%(2)$ & $48.6 \%(35)$ & $P=0.000$ \\
\hline
\end{tabular}

\begin{tabular}{|c|c|c|c|}
\hline & Baseline & End of study & $P$ \\
\hline $\mathrm{PaCO}_{2}-\mathrm{NIV}$ group $(\mathrm{mmHg})$ & $49.2 \pm 1.9$ & $39.8 \pm 2.1$ & 0.001 \\
\hline $\mathrm{PaCO}_{2}$ - Non NIV group ( $\mathrm{mmHg}$ ) & $49.1 \pm 2.1$ & $56.1 \pm 3.7$ & \\
\hline $\mathrm{PaO}_{2}-\mathrm{NIV}$ group $(\mathrm{mm} \mathrm{Hg})$ & $59.3 \pm 3.1$ & $67.8 \pm 3.3$ & 0.00 \\
\hline $\mathrm{PaO}_{2}$ - Non NIV $(\mathrm{mm} \mathrm{Hg})$ & $58.9 \pm 2.8$ & $56.4 \pm 3.8$ & \\
\hline SRI - NIV group & $51.7 \pm 5.3$ & $67.6 \pm 7$ & 0.00 \\
\hline SRI - Non NIV & $50.9 \pm 3.2$ & $55.4 \pm 3.2$ & \\
\hline
\end{tabular}

Table 4: Comparison of FEV1 and exercise tolerance between noninvasive ventilation and control groups

\begin{tabular}{lccc}
\hline & Baseline & $\begin{array}{c}\text { End of } \\
\text { study }\end{array}$ & $\boldsymbol{P}$ \\
\hline FEV1 - NIV (\%) & $42.1 \pm 1.9\left(1^{\text {st }}\right.$ month $)$ & $40.5 \pm 1.1$ & 0.12 \\
FEV1 - Non NIV & $41.2 \pm 2.1\left(1^{\text {st }}\right.$ month $)$ & $36.8 \pm 1.3$ & \\
6MWT (metres) - NIV & $308.2 \pm 30.1\left(1^{\text {st }}\right.$ month $)$ & $304 \pm 26.0$ & 0.20 \\
6MWT - Non NIV & $307.4 \pm 25.4\left(1^{\text {st }}\right.$ month $)$ & $291.3 \pm 18.2$ & \\
\hline
\end{tabular}

clinical research. Measures are being widely implemented to improve management and provide a better quality of life for the patients. Advanced COPD is characterised by irreversible severe airflow obstruction and chronic hypercapnia. Globally, home mechanical ventilation (HMV), mainly noninvasive is increasingly employed to treat patients suffering from chronic hypercapnic respiratory failure. ${ }^{[4]}$ The rapid growth of HMV has been attributed to increased awareness of and experience with the indication and technology; the availability of affordable NIV machines; pressure to reduce hospital stay; and improved life expectancy in treated patients. Compared with older reports, COPD patients constitute an increasingly high proportion of the population on HMV, and a growing number of them are maintained on home NIV. Even though it has been shown to reduce intubation and in-hospital mortality in patients with AHRF, little information exists on the outcomes following discharge. Respiratory muscle fatigue significantly contributes to gas exchange abnormalities in COPD. NIV may help overcome the fatigue by resting the respiratory muscles and thus help improve gas exchange. Patients with COPD are more likely to have nocturnal hypoventilation, especially during rapid eye movement (REM) sleep. This is due to atonia of intercostal muscles and results in decreased chest wall

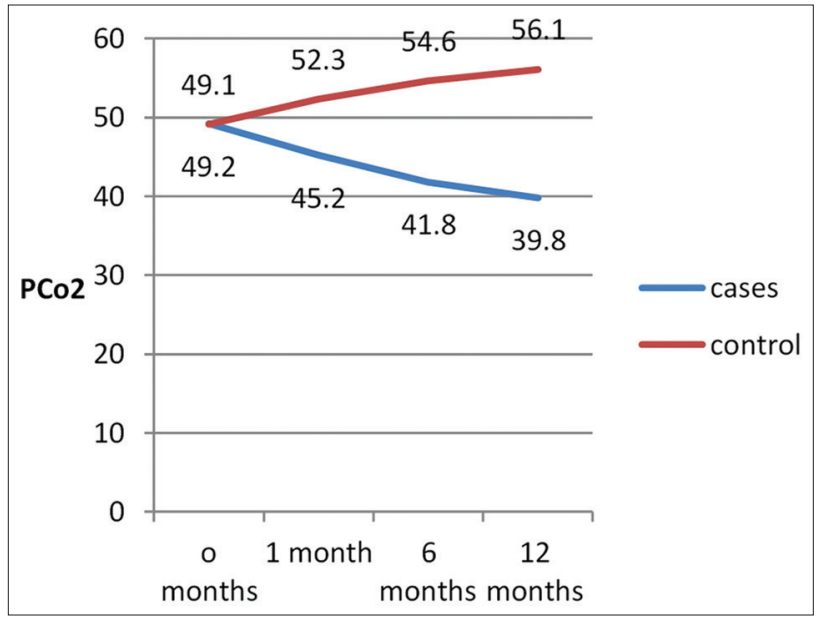

Figure 1: $\mathrm{PaCO}_{2}$ trend over the study period

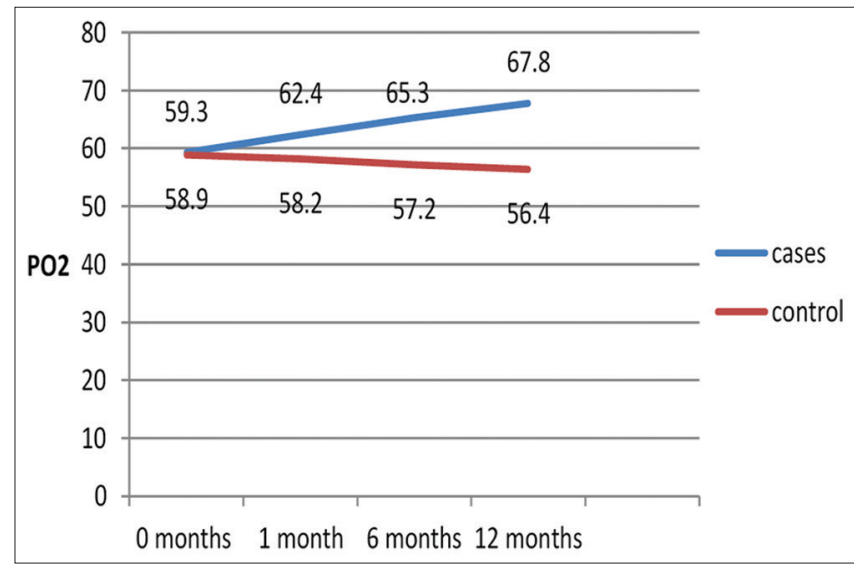

Figure 2: Changes in $\mathrm{PaO}_{2}$ over the study period 
compliance. This atonia results in diaphragm being the only muscle of respiration. Moreover, the hypercapnic ventilatory response is reduced during sleep, especially during REM sleep.

Over the past decade, more and more patients are using home NIV in India along with home oxygen. The demand for home NIV is increasing because of the rising cost of in-patient care in hospitals, availability of compact NIV machines and comfortable masks. However, there are no data from India to support LTNIV use.

In this study, death was taken as the primary endpoint, and secondary endpoints included other patient-centered outcomes. We found that there was a significant reduction in mortality, hospital re-admissions, ICU admissions, AHRF, ventilator requirement and improvement in $\mathrm{PaCO}_{2}, \mathrm{PaO}_{2}$ and SRI score in domiciliary NIV group. By previous studies, we found nosignificant changes in lung function following initiation of LTNIV.

Previous studies reveal conflicting results on home NIV in COPD patients. Earlier small studies showed benefit from home NIV, but the later randomized control trials yielded conflicting results in chronic hypercapnic COPD patients. The discrepancies in the results are due to the difference in criteria in patient selection, use of NIV settings incapable of achieving adequate ventilation, and poor adherence to NIV.

Several outcome measures have been investigated for the use of home NIV in COPD patients. Uncontrolled data have suggested that home NIV might reduce both hospital admission and clinic visits in severe COPD with hypercapnic respiratory failure. Jones et al. administered home NIV to 11 stable COPD patients with chronic hypercapnia who did not respond to conventional treatment. ${ }^{[5]}$ Hospital admissions and clinic visits were halved in the subsequent year, together with a sustained improvement in ABGs. A cost saving was revealed with home NIV in severe COPD in another similar uncontrolled observational study. ${ }^{[6]}$

Conversely, results from randomized controlled trials (RCTs) have been conflicting. There are studies suggesting that home NIV was not superior to standard treatment instable severe COPD. RCT of domiciliary noninvasive positive pressure ventilation in severe COPD by Garrod et al. revealed no significant changes in lung function. ${ }^{[7]}$ Nocturnal NIV in COPD patients with prolonged hypercapnia for acute respiratory failure: randomized, controlled, parallel group study conducted by Struik et al. concluded that there is an improvement in arterial gases, but no effect on survival/ admissions. ${ }^{[8]}$

A more recent study by Cheung et al. tested the hypothesis that continuation of home NIV after an episode of AHRF treated by NIV in COPD patients would reduce the likelihood of recurrent AHRF leading to death or requiring NIV/intubation. ${ }^{[9]}$ The proportion of patients developing recurrent AHRF in the NIV group was $38.5 \%$ versu. $60.2 \%$ at 1 year $(P=0.039)$.
Home NIV was found to reduce mortality in COPD patients with persistent hypercapnia in a study in Germany by Köhnlein et al. This study was conducted in stable COPD patients (no exacerbation in a 4 weeks run in period). 1-year mortality was $12 \%$ in NIV group and $33 \%$ in control group, revealing a clear advantage for home NIV compared to control. This trial had to be terminated early because of significant mortality reduction observed in the NIV group. ${ }^{[10]}$

In contrast, the RESCUE trial from the Netherlands failed to show a survival benefit in patients with home NIV. In this trial, the patients were recruited following an acute exacerbation of COPD with respiratory acidosis requiring NIV support. On discharge from hospital, the patients were randomized to receive either domiciliary NIV or standard care. Even though this study reduced mean noctutnal $\mathrm{PaCO}_{2}$ in the NIV group compared to standard care, there was no effect on survival. ${ }^{[8]}$ This study has a recruitment protocol similar to our study, i.e., patients were recruited following an acute hypercapneic exacerbation of COPD. However, previous history of hypercapnic respiratory failure was not considered as an inclusion criteria. Hence, the recruited patients may not have had significant chronic type II respiratory failure that may have benefited from home NIV.

In another recently published trial by Murphy et al. it was found that adding home NIV to home oxygen therapy prolonged the time to readmission and death. The eligibility criteria for this study were very stringent. Patients were assessed 2 weeks after an episode of resolution of decompensated respiratory acidosis. Persistent hypercapnia $\left(\mathrm{PaCO}_{2}>53 \mathrm{mmHg}\right)$ with $\mathrm{pH}>7.3$, was required to be eligible for the study. This study also adopted a mean inspiratory pressure of $24 \mathrm{~cm} \mathrm{H}_{2} \mathrm{O}$ and EPAP of $4 \mathrm{~cm} \mathrm{H}_{2} \mathrm{O}$. ${ }^{[1]}$

In this study, even though there is mortality benefit as well as improvement in $\mathrm{PaCO}_{2}$, there was no improvement in forced expiratory volume in $1 \mathrm{~s}$ or 6MWD at the end of 1 year study. As all the patients had severe-to-very severe COPD with chronic type II respiratory failure, an improvement in these parameters could not be demonstrated.

The benefits of domiciliary NIV are well documented mainly in patients with neuromuscular disorders. In several studies, participants reported experiencing benefits such as improved breathing, a sense of immediate relief, good sleep, increased alertness, and reduced dyspnea on exertion after LTNIV use. In a study by Ando et al. Regarding acceptance of NIV in patients with motor neurone disease; eventhough, it was found to be effective in respiratory support, many of the patients were not tolerating NIV due to psychological reasons. The threat to the self, the sense of loss of control, and negative views of NIV resulting from anxiety were more important to those patients than prolonging life in its current form. ${ }^{[12]}$ Acceptance was not a problem in our patients; eventhough, the psychological aspects were not analyzed based on structured questionnaire. 
One of the limitations of our study is the small sample size. Even though there was active and control group, it was not a RCT. We could not design an RCT as the acceptability of LTNIV is low in our clinical experience due for financial and social reasons.

\section{Conclusion}

Over the past two decades, noninvasive ventilation has emerged as an indispensable respiratory modality in the management of hypercapnic respiratory failure. In our study, patients tolerated LTNIV well and had better outcome compared to those without NIV. There was significant reduction in mortality, hospital admissions, ICU admissions, AHRF, ventilator requirement, and improvement in $\mathrm{PaCO}_{2}, \mathrm{PaO}_{2}$ and SRI score in domiciliary NIV group. Hence, long-term domiciliary NIV can be considered as a treatment option in those admitted with recurrent type II respiratory failure.

The benefits of LTNIV should be confirmed with multicenter RCT. Since there are no definite guidelines for LTNIV, various trials have adopted various cut-off values for $\mathrm{PaCO}_{2}$ to be eligible for LTNIV. Like the LTOT guidelines, definite protocol should be formulated for initiation, maintenance, and cessation of LTNIV use in patients with recurrent hypercapnic respiratory failure.

\section{Financial support and sponsorship}

Nil.

\section{Conflicts of interest}

There are no conflicts of interest.

\section{RefERENCES}

1. Global Strategy for the Diagnosis, Management, and Prevention of COPD: Global Initiative for Chronic Obstructive Lung Disease; 2017. Available from: http://www.goldcopd.org. [Last accessed on 2017 Dec 28].
2. Galli JA, Krahnke JS, James Mamary A, Shenoy K, Zhao H, Criner GJ, et al. Home non-invasive ventilation use following acute hypercapnic respiratory failure in COPD. Respir Med 2014;108:722-8.

3. Davidson AC, Banham S, Elliott M, Kennedy D, Gelder C, Glossop A, et al. BTS/ICS guideline for the ventilatory management of acute hypercapnic respiratory failure in adults. Thorax 2016;71 Suppl 2:ii1-35.

4. Ankjærgaard KL, Maibom SL, Wilcke JT. Long-term non-invasive ventilation reduces readmissions in COPD patients with two or more episodes of acute hypercapnic respiratory failure. Eur Clin Respir J 2016;3:28303.

5. Jones SE, Packham S, Hebden M, Smith AP. Domiciliary nocturnal intermittent positive pressure ventilation in patients with respiratory failure due to severe COPD: Long-term follow up and effect on survival. Thorax 1998;53:495-8.

6. Tuggey JM, Plant PK, Elliott MW. Domiciliary non-invasive ventilation for recurrent acidotic exacerbations of COPD: An economic analysis. Thorax 2003;58:867-71.

7. Garrod R, Mikelsons C, Paul EA, Wedzicha JA. Randomized controlled trial of domiciliary noninvasive positive pressure ventilation and physical training in severe chronic obstructive pulmonary disease. Am J Respir Crit Care Med 2000;162:1335-41.

8. Struik FM, Sprooten RT, Kerstjens HA, Bladder G, Zijnen M, Asin J, et al. Nocturnal non-invasive ventilation in COPD patients with prolonged hypercapnia after ventilatory support for acute respiratory failure: A randomised, controlled, parallel-group study. Thorax 2014;69:826-34.

9. Cheung AP, Chan VL, Liong JT, Lam JY, Leung WS, Lin A, et al. A pilot trial of non-invasive home ventilation after acidotic respiratory failure in chronic obstructive pulmonary disease. Int J Tuberc Lung Dis 2010;14:642-9.

10. Köhnlein T, Windisch W, Köhler D, Drabik A, Geiseler J, Hartl S, et al. Non-invasive positive pressure ventilation for the treatment of severe stable chronic obstructive pulmonary disease: A prospective, multicentre, randomised, controlled clinical trial. Lancet Respir Med 2014;2:698-705.

11. Murphy PB, Rehal S, Arbane G, Bourke S, Calverley PM, Crook AM, et al. Effect of home noninvasive ventilation with oxygen therapy vs. oxygen therapy alone on hospital readmission or death after an acute COPD exacerbation: A randomized clinical trial. JAMA 2017;317:2177-86.

12. Ando H, Williams C, Angus RM, Thornton EW, Chakrabarti B, Cousins R, et al. Why don't they accept non-invasive ventilation?: Insight into the interpersonal perspectives of patients with motor neurone disease. Br J Health Psychol 2015;20:341-59. 